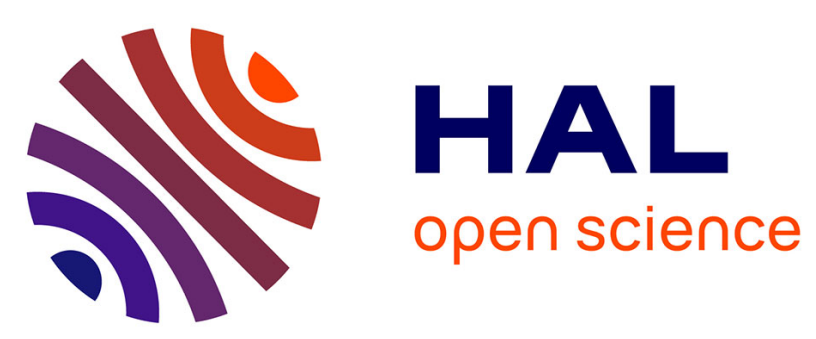

\title{
Residual generator design for singular bilinear systems subjected to unmeasurable disturbances : an LMI approach
}

Michel Zasadzinski, Eric Magarotto, Hugues Rafaralahy, Harouna Souley Ali

\section{- To cite this version:}

Michel Zasadzinski, Eric Magarotto, Hugues Rafaralahy, Harouna Souley Ali. Residual generator design for singular bilinear systems subjected to unmeasurable disturbances: an LMI approach. Automatica, 2003, 39 (4), pp.703-713. 10.1016/S0005-1098(02)00301-1 . hal-00098067

\section{HAL Id: hal-00098067 https://hal.science/hal-00098067}

Submitted on 23 Sep 2006

HAL is a multi-disciplinary open access archive for the deposit and dissemination of scientific research documents, whether they are published or not. The documents may come from teaching and research institutions in France or abroad, or from public or private research centers.
L'archive ouverte pluridisciplinaire HAL, est destinée au dépôt et à la diffusion de documents scientifiques de niveau recherche, publiés ou non, émanant des établissements d'enseignement et de recherche français ou étrangers, des laboratoires publics ou privés. 


\title{
Residual generator design for singular bilinear systems subjected to unmeasurable disturbances : an LMI approach
}

\author{
M. Zasadzinskia ${ }^{a *}$ E. Magarotto ${ }^{b}$, H. Rafaralahy ${ }^{a}$, H. Souley $\operatorname{Ali}^{a}$ \\ ${ }^{a}$ CRAN - CNRS \\ IUT de Longwy, Université Henri Poincaré - Nancy I \\ 186 rue de Lorraine, 54400 Cosnes et Romain, FRANCE \\ ${ }^{b}$ GREYC - CNRS \\ Université de Caen Basse-Normandie \\ 6 boulevard du Marchal Juin, 14050 Caen Cedex, FRANCE
}

\begin{abstract}
In this paper, a method to design a bank of unknown input residual generator (UIRG) for fault detection and isolation is investigated. The plant model is assumed to be a bounded control inputs singular bilinear systems (SBS) subjected to unknown disturbances. The measurements can be bilinear.
\end{abstract}

Keywords : Singular bilinear system, Unknown input residual generator, Exponential stability, Linear matrix inequality.

\section{Introduction}

The theory of observer-based fault detection for non-linear systems has received considerable attention during the last decade. Frank (1993) considered the residual observer for nonlinear systems using a first order approximation, then the stability of the observation error is local. Kinnaert et al. $(1995 ; 1999)$ treated the failure detection and isolation problem for bilinear systems using the regularly persistent observers proposed in (Bornard et al., 1988). This approach is less restrictive than those proposed by Yu and Shields (1996).

This paper is devoted to the design of a bank of UIRG for bounded control inputs SBS subjected to unmeasurable disturbances, without condition on matrix $E^{\#}$. The measurements are bilinear in the control signals. The algebraic part of the generalised state equation and the measurement equation are decomposed according to the failures, the unknown inputs and the bilinearities. The $k^{\text {th }}$ UIRG of the bank is designed in three steps. First, equality constraints are solved to decouple the residual from the unmeasurable disturbances and the $k^{\text {th }}$ failure mode. Second, the problem is converted into a robust stabilisation problem with structured uncertainty, then an LMI approach is used to solve a Lyapunov inequality. Third, the LMI solution is parametrised to solve an algebraic design constraint.

Notations. $\lambda(A)$ is the eigenvalue of the matrix $A, \lambda_{\max }(Q)$ and $\lambda_{\min }(Q)$ are the maximal and the minimal eigenvalues of the symmetric matrix $Q,\|A\|=\sqrt{\lambda_{\max }\left(A^{T} A\right)}\left(\right.$ then $\|x\|=\sqrt{x^{T} x}$ if $x \in \mathbb{R}^{n}$ ) and $\operatorname{sym}(A)=A+A^{T}$.

\section{Problem formulation}

Consider the following time-invariant SBS described by

$$
\begin{aligned}
& E^{\#} \dot{x}=A^{0 \#} x+\sum_{i=1}^{m} u^{i} A^{i \#} x+B^{\#} u+D_{1}^{\#} w+F_{1}^{\#} f \\
& y^{\#}=C^{0 \#} x+\sum_{i=1}^{m} u^{i} C^{i \#} x+D_{2}^{\#} w+F_{2}^{\#} f
\end{aligned}
$$

\footnotetext{
${ }^{*}$ Corresponding author : mzasad@iut-longwy.uhp-nancy.fr
} 
where the state $x \in \mathbb{R}^{n}$, the control input $u \in \mathbb{R}^{m}$ with $u^{T}=\left[u^{1}(t) \cdots u^{m}(t)\right]$, the unknown input $w \in \mathbb{R}^{q}$, the fault $f \in \mathbb{R}^{s}$ and the measured output $y^{\#} \in \mathbb{R}^{p^{\#}}$, and with $E^{\#} \in \mathbb{R}^{r \times n}$ and $\operatorname{rank} E^{\#}=r_{1} \leqslant$ $\min (r, n)$. Note that $u^{i}$ is the $i^{\text {th }}$ component of vector $u$ and $A^{i \#}$ is the matrix associated to $u^{i}$ in the SBS (1). Without loss of generality, $\left[\begin{array}{l}D_{1}^{\#} \\ D_{2}^{\#}\end{array}\right]$ and $\left[\begin{array}{l}F_{1}^{\#} \\ F_{2}^{\#}\end{array}\right]$ are of full column rank, and the control inputs are bounded, i.e. $u \in \Omega \subset \mathbb{R}^{m}$ where

$$
\Omega:=\left\{u \in \mathbb{R}^{m} \mid u_{\min }^{i} \leqslant u^{i} \leqslant u_{\max }^{i} \text { for } i=1, \ldots, m\right\} .
$$

Now, since rank $E^{\#}=r_{1}$, there exists a non-singular matrix $P$ used to extract the algebraic part of (1a), i.e. $P E^{\#}=\left[\begin{array}{c}E \\ 0\end{array}\right], P A^{i \#}=\left[\begin{array}{c}\widetilde{A}^{i} \\ \bar{A}^{i}\end{array}\right], P B^{\#}=\left[\frac{B}{B}\right], P D_{1}^{\#}=\left[\frac{D_{1}}{D_{1}}\right]$ and $P F_{1}^{\#}=\left[\frac{F_{1}}{\bar{F}_{1}}\right]$ with $E \in \mathbb{R}^{r_{1} \times n}$ and $\operatorname{rank} E=r_{1}$. This algebraic part is inserted into the measurement (1b). Thus SBS (1) is restricted system equivalent to

$$
\begin{aligned}
& E \dot{x}=\widetilde{A}^{0} x+\sum_{i=1}^{m} u^{i} \widetilde{A}^{i} x+B u+D_{1} w+F_{1} f \\
& y=C^{0} x+\sum_{i=1}^{m} u^{i} C^{i} x+D_{2} w+F_{2} f
\end{aligned}
$$

where $y=\left[\begin{array}{c}-\bar{B} u \\ y^{\#}\end{array}\right] \in \mathbb{R}^{p}\left(\right.$ with $\left.r_{1}+p=r+p^{\#}\right), C^{i}=\left[\begin{array}{c}\bar{A}^{i} \\ C^{i \#}\end{array}\right], D_{2}=\left[\begin{array}{c}\bar{D}_{1} \\ D_{2}^{\#}\end{array}\right]$ and $F_{2}=\left[\begin{array}{c}\bar{F}_{1} \\ F_{2}^{\#}\end{array}\right]$.

For the SBS (1), the $k^{\text {th }}$ UIRG is described as follows

$$
\begin{aligned}
& \dot{z}=N(u) z+L^{0} y+\sum_{i=1}^{m} u^{i} L^{i} y+G u \\
& \theta^{k}=M(u) z+J y
\end{aligned}
$$

where $z \in \mathbb{R}^{n}$ and $\theta^{k} \in \mathbb{R}\left(\theta^{k}\right.$ is the $k^{\text {th }}$ residual), with $N(u)=N^{0}+\sum_{i=1}^{m} u^{i} N^{i}$ and $M(u)=M^{0}+$ $\sum_{i=1}^{m} u^{i} M^{i}$.

Now the measurement $y$ is decomposed into four parts :

- $y_{1}$ : bilinear, affected by unknown inputs and failures,

- $y_{21}$ : bilinear, only affected by unknown inputs,

- $y_{221}$ : bilinear, free of unknown inputs and failures,

- $y_{222}$ : linear, free of unknown inputs and failures.

First, we make a row compression on matrix $F_{2}$ : there exists a non-singular matrix $V$ such that $V V^{T}=I_{p}$ and $V^{T} F_{2}=\left[\begin{array}{c}F_{21} \\ 0\end{array}\right]$ where $F_{21}$ is of full row rank. Then we have $V^{T} y=\left[\begin{array}{l}y_{1} \\ y_{2}\end{array}\right]\left(\right.$ with $y_{1} \in \mathbb{R}^{p_{1}}$ and $\left.y_{2} \in \mathbb{R}^{p_{2}}\right)$, $V^{T} C^{i}=\left[\begin{array}{c}C_{1}^{i} \\ C_{2}^{i}\end{array}\right]$ and $V^{T} D_{2}=\left[\begin{array}{c}D_{21} \\ D_{22}\end{array}\right]$.

Second, there exists non-singular matrices $V_{1}$ and $V_{2}$ such that $V_{1}^{T} D_{22} V_{2}=\left[\begin{array}{cc}I_{q_{1}} & 0 \\ 0 & 0\end{array}\right]$ and $V_{1} V_{1}^{T}=I_{p_{2}}$. Then we obtain $V_{1}^{T} y_{2}=\left[\begin{array}{l}y_{21} \\ y_{22}\end{array}\right], V_{1}^{T} C_{2}^{i}=\left[\begin{array}{c}C_{21}^{i} \\ C_{22}^{i}\end{array}\right]$ and $\left[\begin{array}{c}w_{1} \\ w_{2}\end{array}\right]=V_{2}^{-1} w$ (with $w_{1} \in \mathbb{R}^{q_{1}}, w_{2} \in \mathbb{R}^{q_{2}}, y_{21} \in \mathbb{R}^{q_{1}}$ and $\left.y_{22} \in \mathbb{R}^{v}\right)$.

Third, there exists a non-singular matrix $V_{3}$ such that $V_{3} V_{3}^{T}=I_{v}$ and $V_{3}^{T}\left[\begin{array}{llll}C_{22}^{1} & \cdots & C_{22}^{m}\end{array}\right]=\left[\begin{array}{ccc}C_{221}^{1} & \cdots & C_{221}^{m} \\ 0 & \cdots & 0\end{array}\right]$ where $\left[\begin{array}{lll}C_{221}^{1} & \cdots & C_{221}^{m}\end{array}\right]$ is of full row rank, which yields with $V_{3}^{T} y_{22}=\left[\begin{array}{l}y_{221} \\ y_{222}\end{array}\right]$ and $V_{3}^{T} C_{22}^{0}=\left[\begin{array}{l}C_{221}^{0} \\ C_{222}^{0}\end{array}\right]$ (with $y_{221} \in \mathbb{R}^{v_{1}}, y_{222} \in \mathbb{R}^{v_{2}}$ and $\left.p=p_{1}+q_{1}+v=p_{1}+q_{1}+v_{1}+v_{2}\right)$.

Defining $A^{i}=\widetilde{A}^{i}-D_{11} C_{21}^{i}$ with $D_{1} V_{2}=\left[\begin{array}{ll}D_{11} & D_{12}\end{array}\right]$ (note that $D_{12}$ is of full column rank) and using the above decompositions, the SBS (3) is equivalent to

$$
\begin{aligned}
E \dot{x} & =A^{0} x+\sum_{i=1}^{m} u^{i} A^{i} x+B u+D_{11} y_{21}+D_{12} w_{2}+F_{11} f_{1} \\
y_{1} & =C_{1}^{0} x+\sum_{i=1}^{m} u^{i} C_{1}^{i} x+D_{21} w+F_{21} f \\
y_{21} & =C_{21}^{0} x+\sum_{i=1}^{m} u^{i} C_{21}^{i} x+w_{1}
\end{aligned}
$$




$$
\begin{aligned}
& y_{221}=C_{221}^{0} x+\sum_{i=1}^{m} u^{i} C_{221}^{i} x \\
& y_{222}=C_{222}^{0} x
\end{aligned}
$$

where the full column rank matrix $F_{11}$ and the fault vector $f_{1}$ are given by $\left[\begin{array}{ll}F_{11} & 0\end{array}\right]=F_{1} U$ and $\left[\begin{array}{l}f_{1} \\ f_{2}\end{array}\right]=U^{T} f$ with $U U^{T}=I_{s} . f_{1}$ is obtained from the column compression on matrix $F_{1}$ and is the part of the fault vector $f$ which can be detected and isolated. Let $\bar{s}$ be the size of $f_{1},\left(F_{11} \in \mathbb{R}^{r_{1} \times \bar{s}}\right.$ with $\left.\operatorname{rank} F_{11}=\bar{s} \leqslant s\right)$. The problem of failure detection and isolation can be treated by using a bank of UIRG. For each residual $\theta^{k}$, decompose the fault vector $f_{1}$ into two subvectors $\bar{f}_{1(k)}$ and $\widetilde{f}_{1(k)}$, the matrix $F_{11}$ is also decomposed into $\bar{F}_{11(k)}$ and $\widetilde{F}_{11(k)}$. To take a decision in a diagnostic procedure, the residual $\theta^{k}$ must be insensitive to the failure modes $\bar{f}_{1(k)}$ and sensitive to the failure modes $\widetilde{f}_{1(k)}$. Then the problem of the generation of residual $\theta^{k}$ can be stated as in the following definition.

Definition 1 (UIRG). The system (4) is a UIRG for the SBS (5) (or (1)) if there exist $N^{i}, L^{i}, G, M^{i}$ and $J$ such that:

i) if $f_{1(k)}=0$, then $\theta^{k}$ decays exponentially to zero,

ii) if $\bar{f}_{1(k)} \neq 0$ and $\tilde{f}_{1(k)}=0$, then $\theta^{k}$ decays exponentially to zero,

iii) if $\widetilde{f}_{1(k)} \neq 0$, then $\theta^{k}$ depends on $\widetilde{f}_{1(k)}$,

hold $\forall w, \forall x_{0}$ and $\forall z_{0}$.

Note that the $k^{\text {th }}$ UIRG (4) can be expressed in terms of notations used in SBS (5) as follows

$$
\begin{aligned}
\dot{z} & =N(u) z+L_{21}^{0} y_{21}+L_{221}^{0} y_{221}+L_{222}^{0} y_{222}+G u+\sum_{i=1}^{m} u^{i}\left(L_{21}^{i} y_{21}+L_{221}^{i} y_{221}+L_{222}^{i} y_{222}\right) \\
\theta^{k} & =M(u) z+J_{221} y_{221}+J_{222} y_{222} .
\end{aligned}
$$

The correspondence between the notations used in (4) and (6) is given by $(i=0, \ldots, m)$

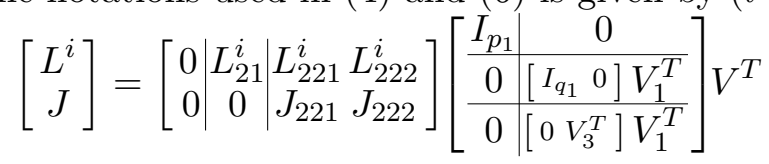

\section{Design of the $k^{\text {th }}$ UIRG}

In this section, the proposed approach for the $k^{\text {th }}$ UIRG design is based on Lyapunov stability. This UIRG has an exponential decay rate and sufficient conditions for its existence are given. When it is no specified, the superscript $i$ stands for $i=0, \ldots, m$. In order to satisfy condition (ii), the subvector $\bar{f}_{1(k)}$ of the vector $f_{1}$ is considered as an unknown input in the $k^{\text {th }}$ UIRG design. Define the error vector $e$ as

$$
e=z-T E x
$$

where $T \in \mathbb{R}^{n \times r_{1}}$, then we have

$$
\begin{array}{r}
\dot{e}=N(u) e+\left(N^{0} T E-T A^{0}+L_{221}^{0} C_{221}^{0}+L_{222}^{0} C_{222}^{0}\right) x+(G-T B) u+\left(L_{21}^{0}-T D_{11}\right) y_{21} \\
-T\left[\begin{array}{ll}
D_{12} & \bar{F}_{11(k)}
\end{array}\right]\left[\begin{array}{c}
w_{2} \\
\bar{f}_{1(k)}
\end{array}\right]+\sum_{i=1}^{m} u^{i}\left(N^{i} T E-T A^{i}+L_{221}^{0} C_{221}^{i}+L_{222}^{i} C_{222}^{0}\right) x-T \widetilde{F}_{11(k)} \widetilde{f}_{1(k)} \\
+\sum_{i=1}^{m} u^{i} L_{21}^{i} y_{21}+\sum_{i=1}^{m} u^{i} L_{221}^{i}\left(C_{221}^{0}+\sum_{j=1}^{m} u^{j} C_{221}^{j}\right) x .
\end{array}
$$

Inserting (8) into (6b), the $k^{\text {th }}$ residual is given by

$$
\theta^{k}=M(u) e+\left(M^{0} T E+J_{221} C_{221}^{0}+J_{222} C_{222}^{0}\right) x+\sum_{i=1}^{m} u^{i}\left(M^{i} T E+J_{221} C_{221}^{i}\right) x .
$$

The following assumption is made in order to ensure that the fault component $\widetilde{f}_{1(k)} \neq 0$ has a non-zero influence on the residual $\theta^{k}$. 
Assumption 1. (Kinnaert et al., 1995; Kinnaert, 1999) For all admissible inputs $u \in \Omega$, the vector fields $T \widetilde{F}_{11(k)} \widetilde{f}_{1(k)}$ are not $(N(u), M(u))$-unobservable.

Using (9) and (10), the solution of the UIRG problem for SBS (5) (or (1)) is given by the following theorem.

Theorem 1. Under Assumption 1, the system (6) (or (4)) is an exponential UIRG for the SBS (5) (or (1)) if there exist $N^{i}, L_{21}^{i}, L_{221}^{i}, L_{222}^{i}, G, M^{i}, J_{221}, J_{222}, T$ and $Q=Q^{T}>0$, and a real $\mu>0$, satisfying the following constraints for all admissible $u \in \Omega$ and for $i=0, \ldots, m$

$$
\begin{aligned}
& N^{i} T E-T A^{i}+L_{221}^{0} C_{221}^{i}+L_{222}^{i} C_{222}^{0}=0 \\
& T\left[D_{12} \quad \bar{F}_{11(k)}\right]=0 \\
& M^{0} T E+J_{221} C_{221}^{0}+J_{222} C_{222}^{0}=0 \\
& M^{i} T E+J_{221} C_{221}^{i}=0 \\
& G=T B \\
& L_{21}^{0}=T D_{11} \\
& L_{21}^{i}=0 \\
& L_{221}^{i}=0 \\
& N^{T}(u) Q+Q N(u)+\mu I<0 .
\end{aligned}
$$

Proof. If constraints (11a)-(11b) and (11e)-(11h) are verified, the error dynamics (9) becomes

$$
\dot{e}=N(u) e-T \widetilde{F}_{11(k)} \widetilde{f}_{1(k)} \text {. }
$$

Let $V(e)=e^{T} Q e$ be a candidate Lyapunov function with $Q=Q^{T}>0$. The time derivative of $V(e)$ along the dynamics $(12)$ with $\widetilde{f}_{1(k)} \equiv 0$, can be expressed as

$$
\dot{V}(e, u)=e^{T}\left(N^{T}(u) Q+Q N(u)\right) e .
$$

If constraint (11i) holds, then the error dynamics (12) is quadratically stable and we have

$$
\dot{V}(e, u)<-\mu e^{T} e \leqslant \frac{-\mu}{\lambda_{\max }(Q)} V(e) \quad \forall u \in \Omega .
$$

Then the error $e(8)$ is exponentially stable, i.e., $\forall u \in \Omega$

$$
\|e(t)\| \leqslant \sqrt{\frac{\lambda_{\max }(Q)}{\lambda_{\min }(Q)}}\left\|z_{0}-T E x_{0}\right\| \exp \left(\frac{-\mu}{2 \lambda_{\max }(Q)} t\right) .
$$

If the constraints (11c) and (11d) are satisfied, the $k^{\text {th }}$ residual (10) can be written as

$$
\theta^{k}=M(u) e .
$$

Then conditions (i) and (ii) in Definition 1 are satisfied. Assumption 1 ensures that the contribution of the non-zero failures $\widetilde{f}_{1(k)}$ on the residual $\theta^{k}$ is different from zero, and the residual $\theta^{k}$ obtained from (12) and (16) fulfills condition (iii) in Definition 1.

Notice that $\dot{V}(e, u)$ is uniformly bounded with respect to $u$ and the UIRG works for non-uniformly observable systems as well as the control input $u$ belongs to $\Omega$, even if $u$ is a non-universal input (see (Bornard et al., 1988)).

The design of the $k^{\text {th }}$ UIRG is decomposed in three parts. First, in section 3.1, the equality constraints (11a)-(11d) in Theorem 1 are solved in order to decouple the error $e$ from the unknown inputs $w$ and the failure component $\bar{f}_{1(k)}$. Second, in section 3.2, the error stability is obtained by solving the inequality constraint (11i) in Theorem 1 using an approach based on an LMI. Third, in section 3.3, a parametrisation is proposed in order to choose, among the solutions of this LMI, those satisfying the decoupling constraints given in section 3.1. 


\subsection{Unknown inputs and failure mode decoupling}

Equations (11c)-(11d) can be equivalently rewritten as

$$
\bar{M} T E+\overline{\bar{J}} \overline{\bar{C}}=0
$$

where matrices $\bar{M}, \overline{\bar{C}}$ and $\overline{\bar{J}}$ are given by

$$
\begin{aligned}
& \bar{M}^{T}=\left[\begin{array}{llll}
M^{0^{T}} & M^{1^{T}} & \ldots & M^{m T}
\end{array}\right], \\
& \overline{\bar{C}}^{T}=\left[\begin{array}{lllll}
C_{221}^{0}{ }^{T} & C_{221}^{1}{ }^{T} & \ldots & C_{221}^{m}{ }^{T} & C_{222}^{0}{ }^{T}
\end{array}\right], \\
& \overline{\bar{J}}=\left[\begin{array}{ccccc}
J_{221} & 0 & \ldots & 0 & J_{222} \\
0 & J_{221} & \ddots & \vdots & 0 \\
\vdots & \ddots & \ddots & 0 & \vdots \\
0 & \ldots & 0 & J_{221} & 0
\end{array}\right] .
\end{aligned}
$$

To solve equation (17) under the constraint (11b), we need to rewrite the latter in terms of $T E$ (see (21) and (22)). Since matrix $E$ is of full row rank, equation

$$
\left[\begin{array}{ll}
D_{12} & \bar{F}_{11(k)}
\end{array}\right]=E \bar{\varphi}
$$

has always a solution given by $\bar{\varphi}=E^{\dagger}\left[D_{12} \bar{F}_{11(k)}\right]$ where $E^{\dagger}$ is any generalised inverse satisfying $E E^{\dagger} E=$ $E$. There exists a non-singular matrix $\bar{U}$ such that $\bar{\varphi} \bar{U}=\left[\varphi_{0}\right]$ where $\varphi$ is of full column rank. We have $E E^{\dagger}=I_{r_{1}}$ since $E$ is of full row rank, then (11b) can be rewritten as

$$
T\left[\begin{array}{ll}
D_{12} & \bar{F}_{11(k)}
\end{array}\right] \bar{U}=T E\left[\begin{array}{ll}
\varphi & 0
\end{array}\right]=0,
$$

or equivalently as

$$
\bar{T} \varphi=0
$$

where

$$
\bar{T}=T E .
$$

A general solution of (21) is given by

$$
\bar{T}=Z_{T}\left(I_{n}-\Phi\right)
$$

where $\Phi=\varphi \varphi^{\dagger}$ and $Z_{T} \in \mathbb{R}^{n \times n}$ is an arbitrary matrix which must be chosen such that equation (22) has a solution $T$. Since $E$ is of full row rank, we have rank $T=\operatorname{rank} \bar{T}$ in relation (22). $Z_{T}$ can be obtained as follows. Choose a non-singular matrix $\overline{\bar{U}}$ such that $E \overline{\bar{U}}=[\bar{E} 0]$ where $\bar{E} \in \mathbb{R}^{r_{1} \times r_{1}}$ and $\operatorname{det} \bar{E} \neq 0$. Then, with the partition of $\bar{T}$ given by $\bar{T} \overline{\bar{U}}=\left[\bar{T}_{1} \bar{T}_{2}\right]$ where $\bar{T}_{2} \in \mathbb{R}^{n \times\left(n-r_{1}\right)}$, matrix $Z_{T}$ is given by $Z_{T}=I_{n}-\bar{T}_{2} \bar{T}_{2}^{\dagger}$, and the unique solution $T$ of (22) is given by

$$
T=Z_{T}\left(I_{n}-\Phi\right) E^{\dagger}
$$

Let $\widetilde{\varphi}_{c}$ be the orthogonal complement of $\varphi$ (Boyd et al., 1994). Since $\left[\varphi\left[\widetilde{\varphi}_{c}\right] \in \mathbb{R}^{n \times n}\right.$ is non-singular, (17) can be equivalently rewritten as

$$
\bar{M} \bar{T}\left[\varphi: \widetilde{\varphi}_{c}\right]+\overline{\bar{J}} \overline{\bar{C}}\left[\varphi: \widetilde{\varphi}_{c}\right]=0
$$

Using (21), equation (25) is equivalent to

$$
\begin{aligned}
& \overline{\bar{J}} \overline{\bar{C}} \varphi=0, \\
& (\bar{M} \bar{T}+\overline{\bar{J}} \overline{\bar{C}}) \widetilde{\varphi}_{c}=0 .
\end{aligned}
$$

Equation (26a) can be equivalently rewritten as

$$
\left[\begin{array}{ll}
J_{221} & J_{222}
\end{array}\right] \widehat{C}=0
$$


where $\widehat{C}=\left[\begin{array}{cccc}C_{221}^{0} \varphi & C_{221}^{1} \varphi & \ldots & C_{222}^{m} \varphi \\ C_{222}^{0} \varphi & 0 & \ldots & 0\end{array}\right]$.

Then the solution of equation (27) is given by

$$
\left[\begin{array}{ll}
J_{221} & J_{222}
\end{array}\right]=\widehat{W}\left(I_{v}-\widehat{C} \widehat{C}^{\dagger}\right)
$$

where $\widehat{W}^{T} \in \mathbb{R}^{v}$ is an arbitrary vector. Using (26a) again, there exists a matrix $\bar{W} \in \mathbb{R}^{(m+1) \times \bar{v}}$ such that

$$
\overline{\bar{J}}=\bar{W}\left(I_{\bar{v}}-\overline{\bar{C}} \varphi(\overline{\bar{C}} \varphi)^{\dagger}\right)
$$

where $\bar{v}=(m+1) v_{1}+v_{2}$. Defining $\bar{P} \in \mathbb{R}^{(m+1) v \times \bar{v}}$ as

$$
\bar{P}=\left[\begin{array}{ccccc}
{\left[\begin{array}{c}
I_{v_{1}} \\
0
\end{array}\right]} & 0 & \ldots & 0 & {\left[\begin{array}{c}
0 \\
I_{v_{2}}
\end{array}\right]} \\
0 & {\left[\begin{array}{c}
I_{v_{1}} \\
0
\end{array}\right]} & \ddots & \vdots & 0 \\
\vdots & \ddots & \ddots & 0 & \vdots \\
0 & \ldots & 0 & {\left[\begin{array}{c}
I_{v_{1}} \\
0
\end{array}\right]} & 0
\end{array}\right]
$$

and using (18c) and (28), $\overline{\bar{J}}$ in (29) can be written as

$$
\overline{\bar{J}}=\bar{W}\left(I_{\bar{v}}-\overline{\bar{C}} \varphi(\overline{\bar{C}} \varphi)^{\dagger}\right)=\left(\widehat{W}\left(I_{v}-\widehat{C} \widehat{C}^{\dagger}\right)\right) \square \bar{P}=\left(\widehat{W} \square I_{(m+1) v}\right)\left(\left(I_{v}-\widehat{C} \widehat{C}^{\dagger}\right) \square \bar{P}\right),
$$

where $\square$ is a block per block product (see appendix A). Since $\left(I_{\bar{v}}-\overline{\bar{C}} \varphi(\overline{\bar{C}} \varphi)^{\dagger}\right)$ is a projector, the solution of (31) is given by

$$
\bar{W}=\left(\widehat{W} \square I_{(m+1) v}\right)\left(\left(I_{v}-\widehat{C} \widehat{C}^{\dagger}\right) \square \bar{P}\right)\left(I_{\bar{v}}-\overline{\bar{C}} \varphi(\overline{\bar{C}} \varphi)^{\dagger}\right)+Z_{W} \overline{\bar{C}} \varphi(\overline{\bar{C}} \varphi)^{\dagger}
$$

where $Z_{W} \in \mathbb{R}^{(m+1) \times \bar{v}}$ is an arbitrary matrix. By using equations (23) and (29), relation (25) becomes

$$
\bar{M} Z_{T}\left(I_{n}-\Phi\right)+\bar{W}\left(I_{\bar{v}}-\overline{\bar{C}} \varphi(\overline{\bar{C}} \varphi)^{\dagger}\right) \overline{\bar{C}}=0 .
$$

Then, inserting (32) into (33) gives

$$
\begin{aligned}
0 & =\left[\begin{array}{ll}
\bar{M} & \widehat{W} \square I_{(m+1) v} \mid Z_{W}
\end{array}\right]\left[\begin{array}{c}
\mathcal{W} \\
0
\end{array}\right] \\
& =\left[\begin{array}{ll}
\bar{M} & \widehat{W} \square I_{(m+1) v}
\end{array}\right] \mathcal{W}
\end{aligned}
$$

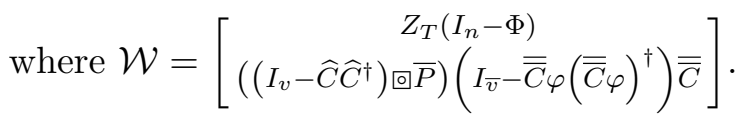

Using the block Kronecker product (Koning et al., 1991), (34) is equivalent to

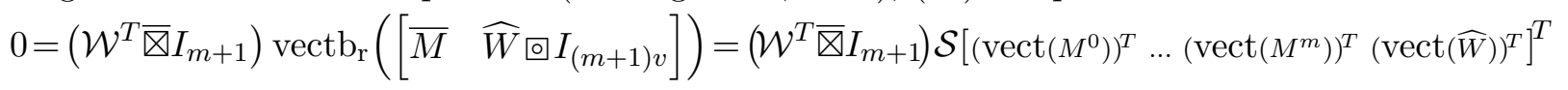

where $\bar{\nabla}$ is the right block Kronecker product (see appendix A) and $\mathcal{S} \in \mathbb{R}^{s_{1} \times s_{2}}$ is a selection matrix (see appendix B for the construction of $\mathcal{S}$ ) with $s_{1}=(m+1)(n+(m+1) v)$ and $s_{2}=v+(m+1) n$. Then $\bar{M}$ and $\widehat{W}$ are given by

$$
\left[\begin{array}{llll}
\left(\operatorname{vect}\left(M^{0}\right)\right)^{T} & \ldots & \left(\operatorname{vect}\left(M^{m}\right)\right)^{T} \quad(\operatorname{vect}(\widehat{W}))^{T}
\end{array}\right]^{T}=\left(I_{v+(m+1) n}-\left(\left(\mathcal{W}^{T} \bar{\nabla} I_{m+1}\right) \mathcal{S}\right)^{\dagger}\left(\mathcal{W}^{T} \bar{\nabla} I_{m+1}\right) \mathcal{S}\right) \mathcal{Z}(36)
$$

where $\mathcal{Z} \in \mathbb{R}^{v+(m+1) n}$ is an arbitrary vector.

Write matrices $N^{i}$ as follows

$$
N^{i}=T A^{i}-K_{1}^{0} C_{221}^{i}-K_{2}^{i} C_{222}^{0} \quad i=0, \ldots, m,
$$


then using (11a), (22) and (23), matrices $L_{221}^{0}$ and $L_{222}^{i}$ satisfy the following equation

$$
\left[\begin{array}{lllll}
L_{221}^{0} & L_{222}^{0} & L_{222}^{1} & \ldots & L_{222}^{m}
\end{array}\right] \Gamma=\Psi
$$

where

$$
\begin{gathered}
\Gamma=\left[\begin{array}{cccc}
C_{221}^{0} & C_{221}^{1} & \ldots & C_{221}^{m} \\
C_{222}^{0} & 0 & \ldots & 0 \\
0 & C_{222}^{0} & \ddots & \vdots \\
\vdots & \ddots & \ddots & 0 \\
0 & \ldots & 0 & C_{222}^{0}
\end{array}\right] \\
\Psi=T\left[\begin{array}{lll}
A^{0} \bar{\Phi} & \ldots & \left.A^{m} \bar{\Phi}\right]
\end{array}\right. \\
+\left[\left(\left[K_{1}^{0} K_{2}^{0}\right]\left[\begin{array}{c}
C_{221}^{0} \\
C_{222}^{0}
\end{array}\right] \bar{T}\right) \ldots\left(\left[K_{1}^{0} K_{2}^{m}\right]\left[\left[\begin{array}{l}
C_{221}^{m} \\
C_{222}^{0}
\end{array}\right] \bar{T}\right)\right]\right. \\
\bar{\Phi}=I_{n}-\bar{T}=I_{n}-Z_{T}\left(I_{n}-\Phi\right) .
\end{gathered}
$$

A solution of equation (38) is given by

$$
L=\left[\begin{array}{lllll}
L_{221}^{0} & L_{222}^{0} & L_{222}^{1} & \ldots & L_{222}^{m}
\end{array}\right]=\Psi \Gamma^{\dagger} .
$$

This solution $L$ is a function of the gains $K_{1}^{0}$ and $K_{2}^{i}$ which are computed in section 3.2 by solving inequality (11i). The existence of $L$ is obtained by using a parametrisation of the solution of inequality (11i). This parametrisation is given in the section 3.3 .

\subsection{Stability analysis via an LMI approach}

The aim of this section is the use of an LMI approach to determine matrices $Q, K_{1}^{0}$ and $K_{2}^{i}$ such that inequality (11i) in Theorem 1 holds. Consider each $u^{i}$ in equation (9) and (10) (or (12) and (16)) as a "structured uncertainty". Note that the definition of the "uncertainty set" $\Omega$ in relation (2) can leads to some conservatism in the use of an LMI approach (Boyd et al., 1994) since, in general case, we have $\left|u_{\text {min }}^{i}\right| \neq\left|u_{\max }^{i}\right|$ with $\left|u_{\min }^{i}\right| \neq 1$ and $\left|u_{\max }^{i}\right| \neq 1$. To overcome this conservatism, each $u^{i}$ is rewritten as follows

$$
u^{i}(t)=\alpha^{i}+\sigma^{i} \varepsilon^{i}(t)
$$

where $\alpha^{i}$ and $\sigma^{i}$ are constant reals given by $(i=1, \ldots, m)$

$$
\alpha^{i}=0.5\left(u_{\max }^{i}+u_{\min }^{i}\right) \text { and } \sigma^{i}=0.5\left(u_{\max }^{i}-u_{\min }^{i}\right)
$$

and $\alpha^{0}=1$ and $\sigma^{0}=0$. Using (41a)-(41b), the new "uncertain" variable $\varepsilon$ is defined as $\varepsilon \in \bar{\Omega} \subset \mathbb{R}^{m}$, where

$$
\bar{\Omega}:=\left\{\varepsilon \in \mathbb{R}^{m} \mid-1 \leqslant \varepsilon^{i} \leqslant 1 \text { for } i=1, \ldots, m\right\} .
$$

Using (41a), equation (12) becomes

$$
\dot{e}=\sum_{i=0}^{m} \alpha^{i} N^{i} e+\sum_{i=1}^{m} \sigma^{i} \varepsilon^{i} N^{i} e-T \widetilde{F}_{11(k)} \widetilde{f}_{1(k)} .
$$

Using (37), the matrix $\bar{N}$ can be defined as

$$
\bar{N}=\sum_{i=0}^{m} N^{i}=\bar{A}-K \bar{C}
$$

with

$$
\begin{aligned}
\bar{A} & =\sum_{i=0}^{m} \alpha^{i} T A^{i}, \\
K & =\left[\begin{array}{lllll}
K_{1}^{0} & K_{2}^{0} & K_{2}^{1} & \ldots & K_{2}^{m}
\end{array}\right], \\
\bar{C} & =\left[\begin{array}{lllll}
\sum_{i=0}^{m} \alpha^{i} C_{221}^{i}{ }^{T} & \alpha^{0} C_{222}^{0}{ }^{T} & \ldots & \alpha^{m} C_{222}^{0}{ }^{T}
\end{array}\right]^{T} .
\end{aligned}
$$


If $\widetilde{f}_{1(k)} \equiv 0$, the insertion of equation (44) into (43) gives

$$
\dot{e}=\left(\bar{N}+H_{1} \Delta(\varepsilon) H_{2}\right) e
$$

where the matrices $H_{1} \in \mathbb{R}^{n \times m n}, \Delta(\varepsilon) \in \mathbb{R}^{m n \times m n}$ and $H_{2} \in \mathbb{R}^{m n \times n}$ are given by

$$
\begin{aligned}
& H_{1}=\left[\begin{array}{lll}
\sigma^{1} N^{1} & \cdots & \sigma^{m} N^{m}
\end{array}\right], \\
& \Delta(\varepsilon)=\operatorname{bdiag}\left(\varepsilon^{i} I_{n}\right) \\
& H_{2}=\left[\begin{array}{lll}
I_{n} & \ldots & I_{n}
\end{array}\right]^{T},
\end{aligned}
$$

where bdiag(.) denotes a block-diagonal matrix. From (42), the matrix $\Delta(\varepsilon)$ in (46) is bounded as

$$
\|\Delta(\varepsilon)\| \leqslant 1 \text {. }
$$

The conditions for the exponential stability of the UIRG are given in the following theorem.

Theorem 2. If the constraints (11a)-(11h) in Theorem 1 hold, the system (6) (or (4)) is an exponential UIRG for the SBS (5) (or (1)) if there exist $Q=Q^{T}>0, S=S^{T}>0, Y$ and a real $\mu>0$, such that the $L M I$

$$
\bar{S}=\left[\begin{array}{c:c}
\operatorname{sym}\left(Q \bar{A}-Y^{T} \bar{C}\right)+H_{2}^{T} S H_{2}+\mu I_{n} & (2,1)^{T} \\
\hdashline \widetilde{A}^{T} T^{T} Q-\widetilde{C}^{T} Y & -S
\end{array}\right]<0
$$

holds where

$$
\begin{aligned}
Y & =K^{T} Q \\
\widetilde{A} & =\left[\begin{array}{llll}
\sigma^{1} A^{1} & \ldots & \sigma^{m} A^{m}
\end{array}\right] \\
\widetilde{C} & =\left[\begin{array}{cccc}
\sigma^{1} C_{221}^{1} & \sigma^{2} C_{221}^{2} & \ldots & \sigma^{m} C_{221}^{m} \\
0 & 0 & \ldots & 0 \\
\sigma^{1} C_{222}^{0} & 0 & \ldots & 0 \\
0 & \sigma^{2} C_{222}^{0} & \ddots & \vdots \\
\vdots & \ddots & \ddots & 0 \\
0 & \ldots & 0 & \sigma^{m} C_{222}^{0}
\end{array}\right]
\end{aligned}
$$

Proof. Assume that the constraints (11a)-(11h) in Theorem 1 hold and that $\widetilde{f}_{1(k)} \equiv 0$, the error dynamics (9) can be rewritten as (46) and the residual $\theta^{k}$ is given by (16).

Let $\bar{V}(e)=e^{T} \bar{Q} e$ be a Lyapunov function candidate with $\bar{Q}=\bar{Q}^{T}>0$. The reconstruction error $e$ is exponentially stable for all $\varepsilon$ in the polytope $\bar{\Omega}$ if the time-derivative of $\bar{V}(e)$ along the trajectory of (46) satisfies

$$
\dot{\bar{V}}(e, \varepsilon)+\bar{\mu} e^{T} e=e^{T}\left(\bar{N} \bar{Q}+\bar{Q} \bar{N}^{T}+H_{1} \Delta(\varepsilon) H_{2} \bar{Q}+\bar{Q} H_{2}^{T} \Delta(\varepsilon) H_{1}^{T}+\bar{\mu} I_{n}\right) e<0
$$

where $\bar{\mu}>0$ is a given real, and $\Delta(\varepsilon)$ given by (47b) satisfies (48). Now defining $Q=\bar{Q}^{-1}$ and choosing a real $\mu>0$ such that $\mu I_{n}>\bar{\mu} Q^{2}$, inequality (51) can be rewritten as the following LMI

$$
\bar{S}=\left[\begin{array}{c:c}
Q \bar{N}+\bar{N}^{T} Q+H_{2}^{T} S H_{2}+\mu I_{n} & Q H_{1} \\
\hdashline H_{1}^{T} Q & -S
\end{array}\right]<0,
$$

by using the Schur Lemma (see (Boyd et al., 1994)), where $S=S^{T}>0$ must be chosen such that (Boyd et al., 1994)

$$
S \Delta(\varepsilon)=\Delta(\varepsilon) S
$$

in order to take the structure of $\Delta(\varepsilon)$ into account. From the structure of $\Delta(\varepsilon)$ given by (47b), relation (53) holds for all matrices $S=S^{T}>0$ of appropriate dimension. Using the notations introduced in (44), (45) and (50), LMI (52) is equivalent to LMI (49). It is easy to see that if LMI (49) holds, then constraint (11i) in Theorem 1 is verified and the error dynamics (12) is exponentially stable. Since LMI (49) has an affine dependence on variables $Q$ and $Y$, then the feasibility and the solution of LMI (49) can be performed with convex optimisation methods (Boyd et al., 1994). 


\subsection{Parametrisation of the solutions of LMI (49)}

Note that matrix $\Psi$ in (39b) is a function of the solutions $Q$ and $Y$ of LMI (49) (see Theorem 2). The problem to be solved can be stated as follows : parametrise the solution $Y$ of LMI (49) in order to obtain a matrix $\Psi$ such that equation (38) has a solution $L$ given by (40).

Define matrices $\bar{\Phi}_{d}=\operatorname{bdiag}(\bar{\Phi}) \in \mathbb{R}^{(m+1) n \times(m+1) n}$ and $\bar{\Psi}$ as follows

$$
\bar{\Psi}=\left[\begin{array}{lll}
T A^{0} & \ldots & T A^{m}
\end{array}\right]-\left[\begin{array}{lll}
\left(K_{1}^{0} C_{221}^{0}+K_{2}^{0} C_{222}^{0}\right) & \ldots & \left(K_{1}^{0} C_{221}^{m}+K_{2}^{m} C_{222}^{0}\right)
\end{array}\right] .
$$

Using (39c) and (54), equation (38) can be rewritten as

$$
\left(\left[\begin{array}{llll}
L_{221}^{0} & L_{222}^{0} & \ldots & L_{222}^{m}
\end{array}\right]-\left[\begin{array}{llll}
K_{1}^{0} & K_{2}^{0} & \ldots & K_{2}^{m}
\end{array}\right]\right) \Gamma=\bar{\Psi} \bar{\Phi}_{d}
$$

and has a solution $L$ if and only if

$$
\operatorname{Im}\left(\bar{\Phi}_{d}^{T} \bar{\Psi}^{T}\right) \subset \operatorname{Im}\left(\Gamma^{T}\right)
$$

Two cases can be considered. First, assume that condition (56) holds, then equation (38) has a solution $L$ given by (40) when the matrix $\Psi$, given by (39b), is computed with the gain matrix $K(45 \mathrm{~b})$ obtained from the solutions $Q$ and $Y$ of LMI (49). In this case, the parametrisation given below is not required. Second, assume that condition (56) does not hold. In this case, equation (38) does not have a solution $L$ when the matrix $\Psi$ is computed with $K$ (45b) obtained from the solutions $Q$ and $Y$ of LMI (49). Then, to solve equation (38), we must find another gain $K$. Since, $K$ depends on the solution $Y$ of LMI (49) (see (50a)), we propose to parametrise the solution $Y$ in order that both LMI (49) holds (i.e. stability condition) and equation (38) has a solution $L$.

In the sequel, we assume that we can not solve equation (38) by using the solutions $Y$ and $Q$ of LMI (49). In other words, the following assumption is made.

Assumption 2. $\operatorname{Im}\left(\bar{\Phi}_{d}^{T} \bar{\Psi}^{T}\right) \not \subset \operatorname{Im}\left(\Gamma^{T}\right)$.

Then, to solve equation (38), a parametrisation of the solution $Y$ of LMI (49) will be introduced. Let $\widehat{Y}$ be a matrix, with the same dimensions as $Y$, such that

$$
\widehat{Y}^{T}\left[\begin{array}{ll}
\bar{C} & \widetilde{C}
\end{array}\right]=0
$$

Then replacing $Y$ by $(Y+\widehat{Y})$ in LMI (49) does not modify matrix $\bar{S}$, i.e. we still have

$$
\bar{S}=\left[\begin{array}{c:c}
\operatorname{sym}\left(Q \bar{A}-(Y+\widehat{Y})^{T} \bar{C}\right)+H_{2}^{T} S H_{2}+\mu I_{n} & (2,1)^{T} \\
\hdashline \widetilde{A}^{T} T^{T} Q-\widetilde{C}^{T}(Y+\widehat{Y}) & -S
\end{array}\right]<0 .
$$

There exist a matrix $\widetilde{Y}$ and a scaling factor $\delta \geqslant 0$ such that the following LMI

$$
\overline{\bar{S}}=\bar{S}-\left[\begin{array}{c:c}
\delta\left(\bar{C}^{T} \widetilde{Y}+\widetilde{Y}^{T} \bar{C}\right) & \delta \widetilde{Y}^{T} \widetilde{C} \\
\hdashline \delta \widetilde{C}^{T} \widetilde{Y} & 0
\end{array}\right] \leqslant \bar{S}<0 .
$$

holds. Define matrices $\widehat{K}, \widetilde{K}$ and $\bar{K}$ as follows

$$
\begin{aligned}
& \widehat{K}=Q^{-1} \widehat{Y}^{T} \\
& \widetilde{K}=Q^{-1} \widetilde{Y}^{T} \\
& \bar{K}=K+\widehat{K}+\delta \widetilde{K}=\left[\begin{array}{lllll}
\bar{K}_{1}^{0} & \bar{K}_{2}^{0} & \bar{K}_{2}^{1} & \ldots & \bar{K}_{2}^{m}
\end{array}\right] .
\end{aligned}
$$

To compute the matrix $\bar{N}$ in equation (44), replace in (45b) the gain $K$ given by (50a) by $\bar{K}$ obtained by (60c). The stability condition (11i) in Theorem 1 is satisfied since LMI (59) holds if LMI (49) is satisfied. Using (60a) and (60b), and inserting (23) into (39b), (38) is equivalent to

$$
(L-\bar{K}) \Gamma=(\bar{\Psi}+\widehat{K} \Gamma+\delta \widetilde{K} \Gamma) \bar{\Phi}_{d} .
$$

Define a matrix $\Lambda$ (with $(m+1) n$ columns as $\left.\bar{\Phi}_{d}\right)$ such that 


$$
\operatorname{Im}\left(\Lambda^{T}\right)=\operatorname{Im}\left(\bar{\Phi}_{d}^{T}\right) \cap \operatorname{Im}\left(\Gamma^{T}\right) \neq 0 .
$$

Then a necessary and sufficient condition to solve equation (61) (i.e. to have a solution $L$ ) is

$$
\operatorname{Im}\left(\bar{\Phi}_{d}^{T}\left(\bar{\Psi}^{T}+\Gamma^{T} \widehat{K}^{T}+\delta \Gamma^{T} \widetilde{K}^{T}\right)\right) \subset \operatorname{Im} \Lambda .
$$

To study the necessary and sufficient condition given by (63), $\bar{\Psi}$ is decomposed using a non-singular matrix $\Sigma$

$$
\Sigma \bar{\Psi}=\left[\begin{array}{ll}
\bar{\Psi}^{T} & \bar{\Psi}_{c}^{T}
\end{array}\right]^{T}
$$

such that (see condition (56))

$$
\operatorname{Im}\left(\overline{\bar{\Psi}}^{T}\right) \cap \operatorname{Im}\left(\overline{\bar{\Psi}}_{c}^{T}\right)=0 \text { and } \operatorname{Im}\left(\bar{\Phi}_{d}^{T} \overline{\bar{\Psi}}^{T}\right) \subset \operatorname{Im}\left(\Gamma^{T}\right) .
$$

Since Assumption 2 holds, we have $\overline{\bar{\Psi}}_{c} \neq 0$.

Using (62)-(65), equation (61) has a solution $L$ if and only if

$$
\left(\left[\begin{array}{ll}
0 & \bar{\Psi}_{c}^{T}
\end{array}\right]^{T}+\Sigma \widehat{K} \Gamma+\delta \Sigma \widetilde{K} \Gamma\right) \bar{\Phi}_{d}=0
$$

Using (57) and (60a), the kernels of $[\bar{C} \widetilde{C}]^{T}$ and $\bar{\Phi}_{d}^{T}$ are given by (where $\chi$ and $\chi_{d}$ are arbitrary matrices)

$$
\begin{aligned}
& \operatorname{ker}\left(\left[\begin{array}{ll}
\bar{C} & \widetilde{C}
\end{array}\right]^{T}\right)=\operatorname{Im}\left(\widehat{K}^{T}\right)=\operatorname{Im}\left(\Theta^{T} \chi^{T}\right), \\
& \operatorname{ker}\left(\bar{\Phi}_{d}^{T}\right)=\operatorname{Im}\left(\Theta_{d}^{T} \chi_{d}^{T}\right),
\end{aligned}
$$

with $\Theta=I_{v_{1}+(m+1) v_{2}}-\left[\begin{array}{ll}\bar{C} & \widetilde{C}\end{array}\right]\left[\begin{array}{ll}\bar{C} & \widetilde{C}\end{array}\right]^{\dagger}$ and $\Theta_{d}=I_{(m+1) n}-\bar{\Phi}_{d} \bar{\Phi}_{d}^{\dagger}$. Using the matrices introduced in (67), equation (66) can be rewritten as

$$
\left[\begin{array}{lll}
\chi & \widetilde{K} & \chi_{d}
\end{array}\right]\left[\begin{array}{c}
\Theta \Gamma \\
\delta \Gamma \\
-\Theta_{d}
\end{array}\right]=\Sigma^{-1}\left[\begin{array}{c}
0 \\
\bar{\Psi}_{c}
\end{array}\right] .
$$

The necessary and sufficient condition to solve (68) is given by

$$
\operatorname{rank}\left[(\Theta \Gamma)^{T}\left|\Gamma^{T}\right| \Theta_{d}^{T}\right]=\operatorname{rank}\left[(\Theta \Gamma)^{T}\left|\Gamma^{T}\right| \Theta_{d}^{T} \mid\left[0 \overline{\bar{\Psi}}_{c}^{T}\right] \Sigma^{-T}\right]
$$

and a solution of equation (68) is given by

$$
\left[\begin{array}{lll}
\chi & \widetilde{K} & \chi_{d}
\end{array}\right]=\Sigma^{-1}\left[\begin{array}{c}
0 \\
\overline{\bar{\Psi}}_{c}
\end{array}\right]\left[\begin{array}{c}
\Theta \Gamma \\
\delta \Gamma \\
-\Theta_{d}
\end{array}\right]^{\dagger}
$$

With $\chi, \chi_{d}$ and $\widetilde{K}$ given by (70), and the gains $K$ (50a) and $\widehat{K}(60 \mathrm{a})$, and using an appropriate choice of $\delta$ (see (59)), the solution of (38) is then obtained by replacing in (45b) $K$ by $\bar{K}$ (60c).

Remark 1 . The above parametrisation can be considered in a particular important case : the bilinear system (1) is not singular and has linear measurements, i.e. $E \in \mathbb{R}^{n \times n}$, $\operatorname{det} E \neq 0$ and $C^{i \#}=0$ for $i=1, \ldots, m$. In this case, if condition

$$
\operatorname{rank} C_{222}^{0}=\operatorname{rank} C_{222}^{0} \varphi
$$

holds, then equation (38) can be reduced to

$$
L_{222}^{i}=K_{2}^{i}+N^{i} \varphi\left(C_{222}^{0} \varphi\right)^{\dagger} \quad i=0, \ldots, m .
$$

and the parametrisation is not needed. But if condition (71) does not hold, assumption 2 must be checked to know if the parametrisation is required since relation (38) can be rewritten as follows

$$
L_{222}^{i} C_{222}^{0}=K_{2}^{i} C_{222}^{0}+N^{i} \varphi \varphi^{\dagger} \quad i=0, \ldots, m
$$

which is an implicit equation in the unknowns $L_{222}^{i}$. 


\section{Illustrative example}

Let us consider the following SBS with bilinear measurements (see (1)) which is affected by one unknown input $w$ and one default $f$ where the matrices of the system are

$$
\begin{aligned}
& E^{\#}=\left[\begin{array}{lll}
1 & 0 & 0 \\
0 & 1 & 0 \\
0 & 0 & 0
\end{array}\right], A^{0 \#}=\left[\begin{array}{ccc}
-5 & 12 & 0 \\
1 & 0.7 & -2 \\
2 & -3 & -7
\end{array}\right], A^{1 \#}=\left[\begin{array}{ccc}
-1 & -1 & 2 \\
2.5 & -1 & 3 \\
0.1 & -3 & 0
\end{array}\right], \\
& A^{2 \#}=\left[\begin{array}{ccc}
2 & 3.5 & 1 \\
0.2 & 1 & 3 \\
0.1 & -1 & 0
\end{array}\right], B^{\#}=\left[\begin{array}{cc}
0 & 1 \\
1 & 0 \\
-1 & -1
\end{array}\right], D^{1 \#}=\left[\begin{array}{l}
52 \\
0 \\
0
\end{array}\right], \\
& D^{2 \#}=\left[\begin{array}{l}
0 \\
0 \\
0
\end{array}\right], F^{1 \#}=\left[\begin{array}{l}
0 \\
8 \\
0
\end{array}\right], F^{2 \#}=\left[\begin{array}{l}
0 \\
0 \\
0
\end{array}\right], \\
& C^{0 \#}=\left[\begin{array}{lll}
1 & 1 & 0 \\
1 & 0 & 1 \\
0 & 0 & 1
\end{array}\right], C^{1 \#}=\left[\begin{array}{lll}
1 & 0 & 0 \\
0 & 0 & 0 \\
0 & 0 & 0
\end{array}\right], C^{2 \#}=\left[\begin{array}{lll}
2 & 0 & 0 \\
0 & 0 & 0 \\
0 & 0 & 0
\end{array}\right] .
\end{aligned}
$$

The control inputs are bounded as follows (see (2))

$$
u_{\min }^{1}=-3, \quad u_{\max }^{1}=3, \quad u_{\min }^{2}=-1, \quad u_{\max }^{2}=1 .
$$

After the decompositions described in section 2, we get the following SBS (see (5))

$$
\begin{aligned}
& E=\left[\begin{array}{lll}
1 & 0 & 0 \\
0 & 1 & 0
\end{array}\right], A^{0}=\left[\begin{array}{ccc}
-5 & 12 & 0 \\
1 & 0.7 & -2
\end{array}\right], A^{1}=\left[\begin{array}{ccc}
-1 & -1 & 2 \\
2.5 & -1 & 3
\end{array}\right], F_{11}=\left[\begin{array}{l}
0 \\
8
\end{array}\right], \\
& A^{2}=\left[\begin{array}{lll}
2 & 3.5 & 1 \\
0 & 1 & 3
\end{array}\right], B=\left[\begin{array}{ll}
0 & 1 \\
1 & 0
\end{array}\right], D_{12}=\left[\begin{array}{c}
52 \\
0
\end{array}\right], C_{221}^{0}=\left[\begin{array}{ccc}
2 & -3 & -7 \\
1 & 1 & 0
\end{array}\right] \text {, } \\
& C_{221}^{1}=\left[\begin{array}{ccc}
0.1 & -3 & 0 \\
1 & 0 & 0
\end{array}\right], C_{221}^{2}=\left[\begin{array}{ccc}
0.2 & -1 & 0 \\
2 & 0 & 0
\end{array}\right], C_{222}^{0}=\left[\begin{array}{lll}
1 & 0 & 1 \\
0 & 0 & 1
\end{array}\right] \text {. }
\end{aligned}
$$

Notice that $y_{1}, y_{21}$ and $D_{11}$ are empty in this example.

Choosing $\bar{U}=\left[\begin{array}{ll}1 & 0 \\ 0 & 1\end{array}\right]$ and $\overline{\bar{U}}=\left[\begin{array}{ccc}1 & -1 & -1 \\ 0 & 1 & 1 \\ 0 & 0 & 1\end{array}\right]$, and taking $\mu=10^{-2}$, we get as solutions to the LMI (49)

$$
\begin{aligned}
Q= & {\left[\begin{array}{ccc}
1337384.3 & 1651.1387 & 1542.3736 \\
1651.1387 & 668883.55 & 667234.9 \\
1542.3736 & 667234.9 & 669355.82
\end{array}\right], \quad S=5 I_{6}, } \\
Y= & {\left[\begin{array}{ccc}
17.224282 & 261.4016 & -336.19197 \\
0 & 931341.11 & 0 \\
653097.2 & -806240.67 & 1 \\
-633467.38 & -265840.18 & 1155928.1 \\
134.17052 & -929306.33 & -2617.3595 \\
28.841755 & 931779.54 & -563.65639 \\
-3.3460855 & -1862734.7 & 66.974164 \\
166.35836 & 1865207.9 & -3247.99
\end{array}\right] }
\end{aligned}
$$

We deduce the gain $K$ from (50a). Then, after computing $\Gamma, \bar{\Phi}, \Phi_{d}$ and $\bar{\Psi}$ using (39a), (39c) and (54), we remark that Assumption 2 holds. So the parametrisation described in section 3.3 is needed to solve equation (38). The new matrix $\bar{Y}$, which is obtained by computing the solutions $\widehat{Y}$ and $\widetilde{Y}$ of the LMI (59), is given by $\bar{Y}=Y+\widehat{Y}+\widetilde{Y}=Q \bar{K}$

$$
\bar{Y}=\left[\begin{array}{ccc}
17.27087 & 261.2787 & -336.26988 \\
-917914.08 & 2032649 & 180359.88 \\
1602313.2 & -1485682.4 & -838290.34 \\
-706149.45 & 223886.96 & 1515697.3 \\
918047.93 & -2030613 & -182977.03 \\
-917887.68 & 2033046.6 & 179847.63 \\
1835824.6 & -4065349.9 & -360652.44 \\
-1835589.9 & 4068908.8 & 356074.46
\end{array}\right]
$$


and then matrices of the UIRG (6) can be deduced by using relations given above.

Figure 1 shows the error with a good convergence rate as well as Figure 2 shows the convergence of the residual. In Figures 2 and 3 , the failure $f$ appears at $t=15 \mathrm{~s}$ and disappears at $t=15.5 \mathrm{~s}$, while the unmeasurable disturbance $w$ appears at $t=18 \mathrm{~s}$ and disappears at $t=18.3 \mathrm{~s}$. Figure 2 shows that the residual $\theta$ is sensitive to $f$ and insensitive to $w$. This is well seen in Figure 3 where a zoom has been done on the occurence time window. So the designed UIRG can be efficiently used to detect default which appears on a SBS.

\section{Conclusion}

The objective of this paper is the design of a bank of UIRG for SBS subjected to unmeasurable disturbances and with bounded control inputs and bilinear measurements. Sufficient condition is given in order to obtain disturbance decoupled residuals. Considering the bounded control inputs as "structured uncertainties", the exponential stability of the $k^{\text {th }}$ UIRG of the bank is guaranteed by solving an LMI associated to a robust stabilisation problem. A control inputs change of coordinates has been used to reduce the conservatism inherent to the robust control theory. To take algebraic design constraints into account, a parametrisation has been introduced to solve this LMI. The use of this parametrisation is conditioned by a relationship given in Assumption 2.

\section{A Appendix : Right block Kronecker product}

The right block Kronecker product $(A \bar{\nabla} B)$ is defined as follows (Koning et al., 1991)

$$
A \bar{\nabla} B=\left[\begin{array}{ccc}
A \otimes B_{11} & \ldots & A \otimes B_{1 p} \\
\vdots & \ddots & \vdots \\
A \otimes B_{q 1} & \ldots & A \otimes B_{q p}
\end{array}\right]
$$

where $B_{i j}$ denotes the $(i, j)^{\text {th }}$ block of partitioned matrix $B$ and $(A \otimes B)$ is the Kronecker product. The two following relations

$$
\begin{aligned}
& (A \bar{\nabla} B)(C \bar{\nabla} D)=(A C) \bar{\otimes}(B C) \\
& \operatorname{vectb}_{\mathrm{r}}(A X B)=\left(B^{T} \bar{\nabla} A\right) \operatorname{vectb}_{\mathrm{r}}(X)
\end{aligned}
$$

hold, where the operators vect(.) (matrix vectorization) and vectb $\mathrm{r}_{\mathrm{r}}(\cdot)$ (partitioned matrix vectorization per block) are defined as

$$
\operatorname{vect}\left(\left[\begin{array}{ll}
a & b \\
c & d
\end{array}\right]\right)=\left[\begin{array}{l}
a \\
c \\
b \\
d
\end{array}\right], \operatorname{vectb}_{\mathrm{r}}\left(\left[\begin{array}{ll}
A & B \\
C & D
\end{array}\right]\right)=\left[\begin{array}{c}
\operatorname{vect}(A) \\
\operatorname{vect}(B) \\
\operatorname{vect}(C) \\
\operatorname{vect}(D)
\end{array}\right] .
$$

The block per block product $(A \square B)$ is defined as follows

$$
A \boxminus B=\left[\begin{array}{ccc}
A B_{11} & \ldots & A B_{1 p} \\
\vdots & \ddots & \vdots \\
A B_{q 1} & \ldots & A B_{q p}
\end{array}\right],
$$

and has the following property

$$
(A C) \square B=\left(A \square I_{\alpha q}\right)(C \square B)
$$

where $B$ is partitioned in $q \times p$ blocks

$$
B=\left[\begin{array}{ccc}
B_{11} & \ldots & B_{1 p} \\
\vdots & \ddots & \vdots \\
B_{q 1} & \ldots & B_{q p}
\end{array}\right]
$$

and $I_{\alpha q}$ is partitioned in $q \times q$ blocks where $\alpha$ is the number of columns of matrix $A$. 


\section{B Appendix : Selection matrix $\mathcal{S}$ in relation $(35)$}

For example, if $m=2$, the vector vectb $\left(\left[\begin{array}{lll}\bar{M} & \widehat{W} \\ \square & I_{3 v}\end{array}\right]\right)$ in relation (35) is computed as follows

$$
\operatorname{vectb}_{\mathrm{r}}\left(\left[\bar{M} \mid \widehat{W} \square I_{3 v}\right]\right)=\underbrace{\left[\begin{array}{cccc}
I_{n} & 0 & 0 & 0 \\
0 & 0 & 0 & I_{v} \\
0_{2 v \times n} & 0 & 0 & 0 \\
0 & I_{n} & 0 & 0 \\
0_{v \times n} & 0 & 0 & 0 \\
0 & 0 & 0 & I_{v} \\
0_{v \times n} & 0 & 0 & 0 \\
0 & 0 & I_{n} & 0 \\
0_{2 v \times n} & 0 & 0 & 0 \\
0 & 0 & 0 & I_{v}
\end{array}\right]}_{\mathcal{S}} \text { } \begin{array}{c}
\operatorname{vect}\left(M^{0}\right) \\
\operatorname{vect}\left(M^{1}\right) \\
\operatorname{vect}\left(M^{2}\right) \\
\operatorname{vect}(\widehat{W})
\end{array}] \text {. }
$$

\section{References}

Bornard, G., N. Couenne and F. Celle (1988). Regularly persistent observers for bilinear systems. In J. Descusse, M. Fliess, A. Isidori and D. Leborgne (Eds.). 'New Trends in Nonlinear Control Theory'. Vol. 122 of Lecture Notes in Control and Information Sciences. Springer-Verlag. pp. 130-140.

Boyd, S., L. El Ghaoui, E. Féron and V. Balakrishnan (1994). Linear Matrix Inequality in Systems and Control Theory. SIAM. Philadelphia.

Frank, P. (1993). Advances in observer-based fault diagnosis. In 'Proc. Int. Conf. Fault Diagnosis TOOLDIAG'. Toulouse, France.

Kinnaert, M. (1999). 'Robust fault detection based on observers for bilinear systems'. Automatica 35, 1829-1842.

Kinnaert, M., Y. Peng and H. Hammouri (1995). The fundamental problem of residual generation for bilinear systems up to output injection. In 'Proc. European Contr. Conf.'. Rome, Italy.

Koning, R., H. Neudecker and T. Wansbeek (1991). 'Block Kronecker products and the vecb operator'. Lin. Alg. \& Applic. 149, 165-184.

Yu, D. and D.N. Shields (1996). 'A bilinear fault detection observer'. Automatica 32, 1597-1602.

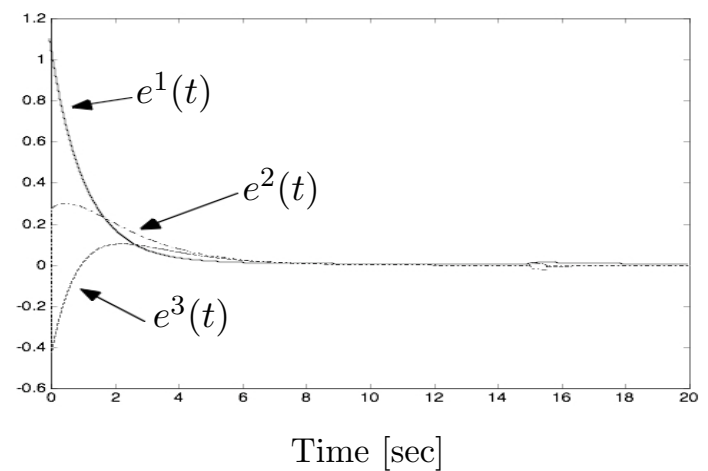

Figure 1: Reconstruction error $e(t)$. 


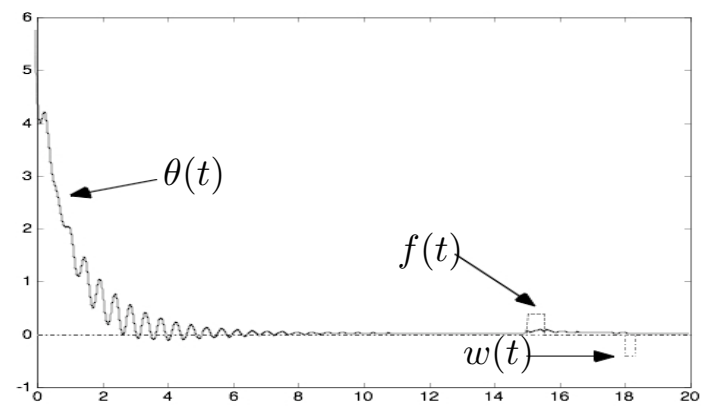

Time $[\mathrm{sec}]$

Figure 2: Residual $\theta(t)$, failure $f(t)$ and disturbance $w(t)$.

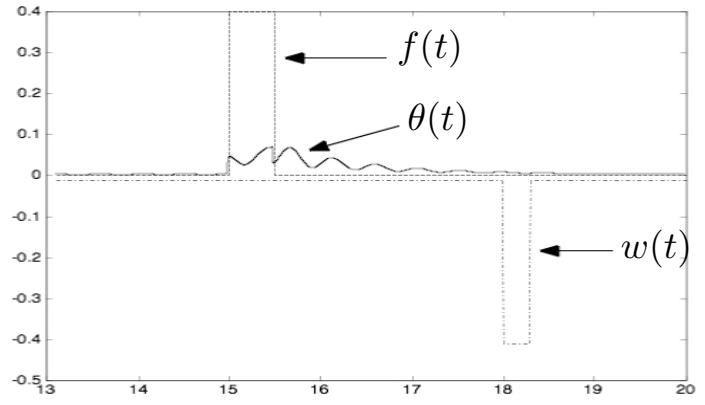

Time [sec]

Figure 3: Zoom on figure 2. 